\title{
FRACTIONATION OF THE SOLAR WIND
}

\author{
R. VON STEIGER \\ Physikalisches Institut, Sidlerstrasse 5, CH-3012 Bern, Switzerland
}

The composition of the solar wind (SW) is not a true sample of the solar composition, but it is fractionated with respect to the solar photosphere. This fractionation follows the well-known first ionization potential (FIP) pattern: When plotting the $\mathrm{SW}$ abundances with respect to the solar abundances as a function of FIP, a step function is obtained (Fig. 1). The step at $\sim 10 \mathrm{eV}$ has a height of 3-5 in the slow SW, but this is reduced to $1.5-2$ in the fast streams, which originate in the coronal holes. The data given in Fig. 1 are collected and discussed in von Steiger \& Geiss (1994), including the "FIP exceptions", Kr and Xe.

The process leading to the observed overabundance of the low-FIPs has been located to operate by atom-ion separation across magnetic field lines in the chromosphere (Geiss, 1982), because this is the only region of the solar atmosphere where a significant fraction of the gas is neutral. The fractionated abundances observed in the $\mathrm{SW}$ are thus important tracers for processes and conditions at this site.

As basic ingredients, every model of the FIP fractionation must treat (1) the ionization and (2) the separation of the gas under chromospheric conditions. Table 1 collects the existing models and indicates by what processes the ionization and the separation are achieved, and whether they are worked out in sufficient detail as to compare their results to the measured data. The FIP models are discussed further in the reviews by von Steiger \& Geiss (1994) and Meyer (1993). Whereas many models use the solar UV as the mode of ionization, many different possibilities have been tried for the mode of separation. As yet, no model is without shortcomings and can claim to fully explain the FIP effect. Since the amplitude of the FIP fractionation is correlated to the SW type (Fig. 1), we believe that integrated chromosphere-corona-SW models are needed to make significant progress. 
TABLE 1. Overview of existing FIP fractionation models

\begin{tabular}{lllc}
\hline Reference & Ionization & Separation & Pred? \\
\hline Vauclair \& Meyer (1985) & - & Gravit. settlement & No \\
Geiss \& Bochsler (1985) & UV Photons & - & No \\
von Steiger \& Geiss (1989) & UV Photons & $\begin{array}{l}\text { Density gradient across B- } \\
\text { field, gravity }\end{array}$ & Yes \\
Hénoux \& Somov (1992) & $?$ & DC currents in B-Field & No \\
Antiochos (1990) & Heat flux from corona & Thernoelectric field & No \\
Marsch et al. (1994) & UV Photons & Diffusion layer & Yes \\
Vauclair (1994) & - & Rising B-field & Yes \\
Tagger et al. (1994) & $?$ & Waves in B-field & No \\
\hline
\end{tabular}

\section{References}

Antiochos, S. K. (1990) Eos Trans. AGU 71(17), 587

Geiss, J. (1982) Space Sci. Rev. 33, 201

Geiss, J., \& Bochsler, P. (1985) in: Rapports Isotopiques dans le Système Solaire, Toulouse: Cepadues-Editions, pp. 213-228

Hénoux, J.-C., \& Somov, B. V. (1992) ESA SP-348, 325-330

Marsch, E., von Steiger, R., \& Bochsler, P. (1994) submitted to $A \& A$

Meyer, J.-P. (1993) in: Prantzos, N. et al. (eds), Origin and Evolution of the Elements, Cambridge Univ. Press, pp. 26-62

Tagger, M., Shukorov, A. M., \& Falgarme, E. (1994) A\&A, in preparation

Vauclair, S. (1994) submitted to $A \& A$

Vauclair, S., \& Meyer, J.-P. (1985) in: Proc. 19th Internat. Cosmic Ray Conf., p. 230

von Steiger, R., \& Geiss, J. (1989) A\&A 225, 222-238

von Steiger, R., \& Geiss, J. (1994) in: Jokipii, J. R. et al. (eds), Cosmic Winds and the Heliosphere. Tucson: University of Arizona Press, submitted 


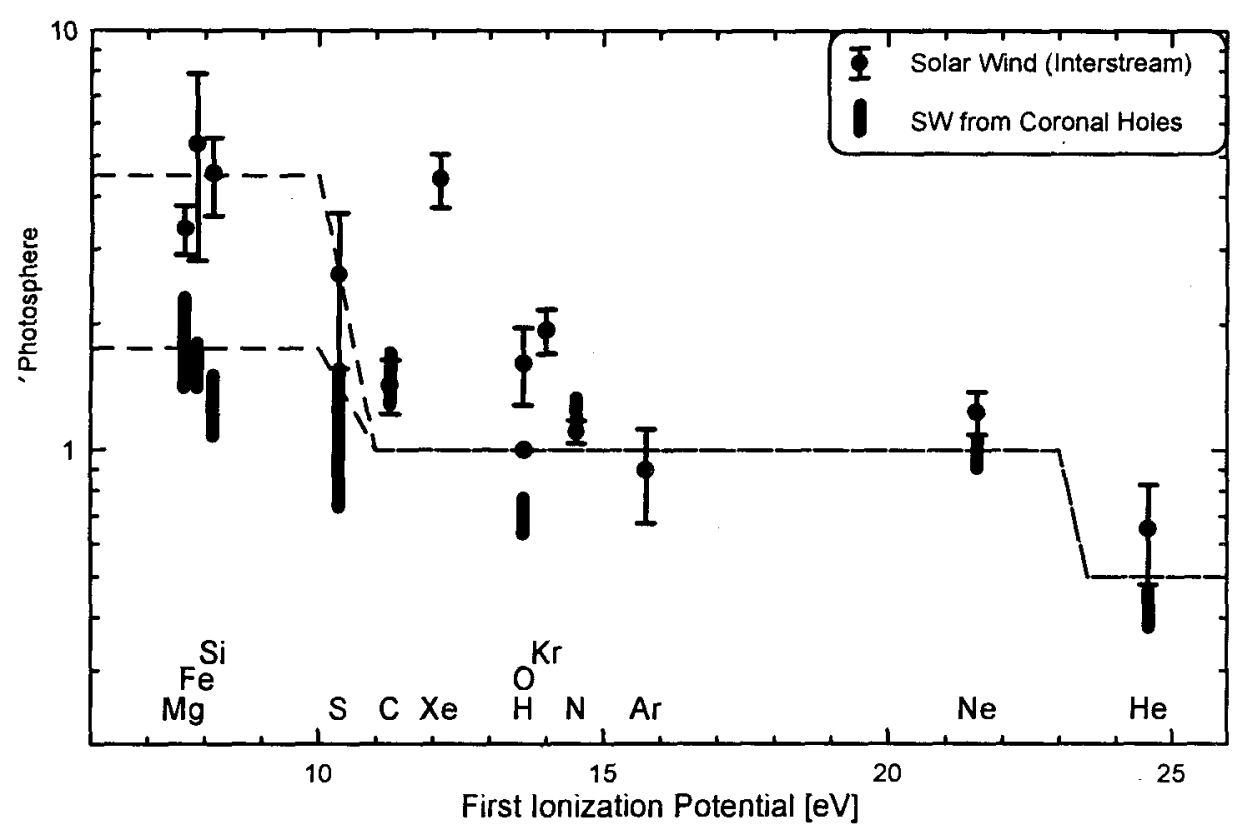

Figure 1. Abundance ratios of all elements measured in the solar wind with respect to $\mathrm{O}$, in relation to the photospheric abundance ratios, plotted $v s$. the FIP. Low-FIP $(<10 \mathrm{e} \mathrm{V)}$ elements are enriched by a factor of 3-5 in the slow SW (as they are in the solar energetic particles), but this enrichment is reduced to a factor of $1.5-2$ in fast streams [cf. von Steiger \& Geiss (1994) for references to and a discussion of the data; the error bars include both the natural variability and the measurement uncertainty]. 